\title{
ANdrée Tretiakoff \\ RESULTS OBTAINED WITH A NEW METHOD \\ FOR THE AUTOMATIC ANALYSIS OF \\ SENTENCE STRUCTURES
}

We present in this paper a method for the automatic analysis of sentence structures.

Our purpose is to constitute a frequency dictionary of the different structures used in the language. This dictionary will enable us to select the most useful sentence structures in order to recommend their exclusive use for the writing of texts intended for automatic translation.

We think that the automatic translation will be possible only if the texts are submitted to rules which limit the complexity of their syntax. These limitations will be the less noticed by an author as only the most unusual structures would have been left out. Of course the number of permitted structures will increase as the automatic translation codes are improved.

The sentence structures are obtained by a statistical analysis of the word strings according to procedures developed in the information theory.

In the present paper we have analysed only groups of two consecutive words as an example of our method.

The same type of analysis can be generalized by considering nonconsecutive words and groups of more than two words.

\section{GROUPS}

The first step of the analysis is to put the words into groups according to their grammatical properties, for example: noun, adjective, article and so on. The number of groups has been limited to keep significative frequencies with respect to the length of the corpus $(3500$ words). In the text under study, we have used 67 groups. A list of these groups is given in Table 3 . 
Of course, our classification is somewhat arbitrary as it is based on a preliminary knowledge of the language. We will show later how the results of the analysis can help us to detect inadequate classifications.

Each word of the corpus has been replaced by a symbol (two figures integer) representing its grammatical group. We consider the words inside the sentence, that is to say between two strong punctuation signs (. ; ! ?). Inside the sentence all punctuation signs are suppressed.

We will call now "words" these symbols.

\section{DICTIONARY OF STRINGS}

The second step is the constitution of a string dictionary.

A sentence containing $N$ words produces $(N-1)$ strings. For instance, the sentence Her daughter gave me an Italian lesson every day represented by the string “ 550401444505048504 ”, produces the following strings:

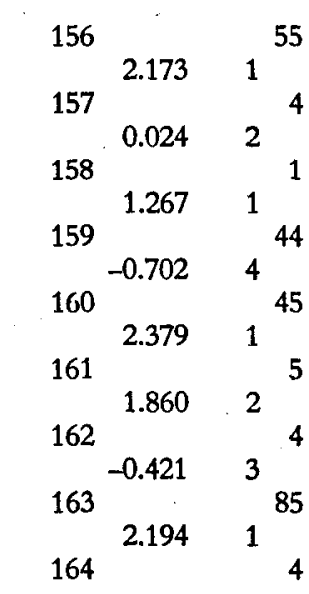

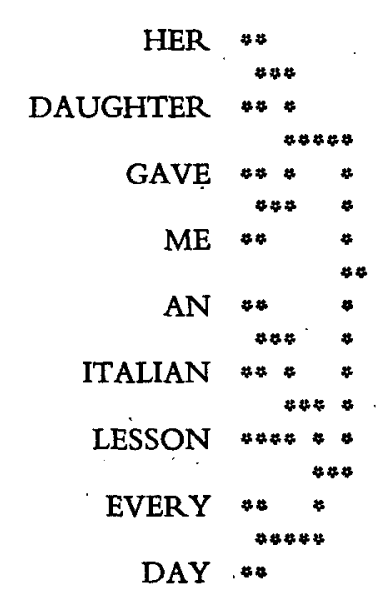
First (complete) string Second string etc...
$\begin{array}{lllllllll}55 & 04 & 01 & 44 & 45 & 05 & 04 & 85 & 04\end{array}$
$\begin{array}{llllllll}04 & 01 & 44 & 45 & 05 & 04 & 85 & 04\end{array}$
$\begin{array}{lllllll}01 & 44 & 45 & 05 & 04 & 85 & 04\end{array}$ $\begin{array}{llllll}44 & 45 & 05 & 04 & 85 & 04\end{array}$ $\begin{array}{lllll}45 & 05 & 04 & 85 & 04\end{array}$ $\begin{array}{llll}05 & 04 & 85 & 04\end{array}$
$\begin{array}{lll}04 & 85 & 04\end{array}$

Last strng . . . . . . . . . . . . 8504

Each string is obtained by suppressing the first word of the preceding string. 
The dictionary emphasizes the identical strings whatever their position in the sentence might be. A sample of the dictionary is given in Table 1.

For example, the string 0504 which means an adjective followed by a common noun at the end of a sentence has the rank number 244 , occurs 9 times in the sentences number $9,10,35$ and so on.

All the strings beginning by the groups 05,04 are also listed.

\section{SENTENCE STRUCTURE}

The last step of the analysis is the production of sentence structures, using the correlations between two consecutive words.

We can compare the probability $P_{j}$ of a word $j$ in the corpus and the conditional probability $P_{j}$ (if $i$ ) of the same word when the preceding word is given equal to $i$. We shall call in this paper " degree of correlation" the logarithm of the ratio of the conditional probability and the probability:

$$
C_{i j}=\log _{2} P_{j}(\text { if } i) / P_{j}
$$

The degree of correlation will be positive when the probability to get a word is increased by the knowledge of the preceding word, and negative when this probability is decreased. It is a measure of the " affinity" of two consecutive words.

This procedure can be generalized by considering groups of more than two words, not necessarily consecutive.

For each sentence of the corpus we can build a structure based on the correlation between two consecutive words in the following way. Inside the sentence, consecutive words are connected two by two in order of decreasing degree of correlation. For instance in the sentence:

$$
\text { She loved a good laugh }
$$

we have the following degrees of correlation:

$$
\begin{aligned}
& \text { She loved }=2.56 \\
& \text { loved a }=1.23 \\
& \text { a good }=2.38 \\
& \text { good laugh }=1.86
\end{aligned}
$$


Therefore the first words to be connected are She and loved then $a$ and good. We will consider that their union is the first level. Then the word laugh will be connected to the group a good. This union will be a second level and finally the two halves of the sentence are connected and this union will be the third level.

This structure can be represented by the following graph, automatically produced by the computer, and by the string 1312 obtained by writing the sequence of the successive levels.

\section{SENTENCE NO 9}

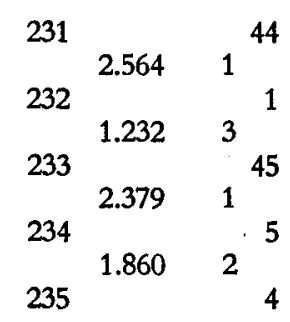

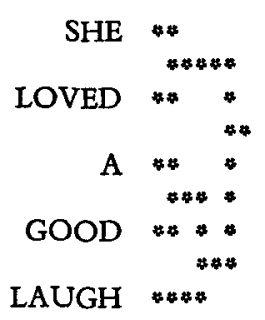

Degrees of correlation:

$$
\begin{aligned}
& \text { She loved. . . . . } 2.56 \\
& \text { loved } a \text {. . . . . } 1.23 \\
& \text { a good . . . . . . . } 2.38 \\
& \text { good laugh . . . . . } 1.86 \\
& \text { String of groups: } 44 \quad 01 \quad 45 \quad 05 \quad 04 \\
& \text { String of levels: } \quad \begin{array}{lllll} 
& 3 & 1 & 2
\end{array}
\end{aligned}
$$

\section{DICTIONARY OF STRUCTURES}

This procedure has been applied for all the sentences of the text, producing strings of numbers which represent the structure of these sentences.

For each string of numbers, by suppressing the highest number we obtain 2 strings representing 2 substructures of this sentence. We carry on this procedure till the string has only 1 number, that is to say represents the structure of a group of 2 words.

For instance the structure of the sentence Her daughter gave me an Italian lesson every day is represented by the following string of numbers: 
SENTENCE NO 5

\begin{tabular}{|c|c|c|c|c|}
\hline 156 & 2.173 & $1^{55}$ & HER & $4 \%$ \\
\hline 157 & & 4 & DAUGHTER & $* *$ \\
\hline & 0.024 & 2 & & 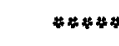 \\
\hline 158 & 1.267 & $1^{1}$ & GAVE & $\begin{array}{c}40 \% \\
74 \%\end{array}$ \\
\hline 159 & -0.702 & 44 & ME & $* *$ \\
\hline 160 & 2.379 & 45 & AN & $* 4$ \\
\hline 161 & 1.860 & $2^{5}$ & ITALIAN & $\# * 4$ \\
\hline 162 & -0.421 & $3^{4}$ & LESSON & $\begin{array}{r}* 4 * 4 \% \\
* 48\end{array}$ \\
\hline 163 & 2.194 & $1^{85}$ & EVERY & 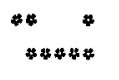 \\
\hline 164 & & 4 & DAY & $*$ \\
\hline
\end{tabular}

\begin{tabular}{|c|c|c|c|c|c|c|c|}
\hline Complete string: & 1 & & - & & & & \\
\hline 1 substring : & & & & & & & \\
\hline 2 substrings: & 1 & 2 & 1 & & 1 & & \\
\hline 3 substrings: & 1 & & 1 & & 1 & & \\
\hline
\end{tabular}

All the structures and substructures are classified in a dictionary, giving their frequencies and the positions of the sentences containing the corresponding word strings (Table 2).

For example, the structure $\begin{array}{llllll}1 & 4 & 2 & 1 & 3 & \text { has the rank number }\end{array}$

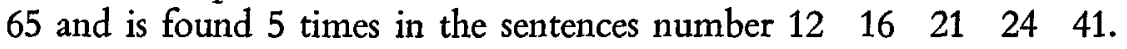

\section{ClASSIFICATION ERRORS}

If the structure of a sentence is unsatisfactory, this can be due to an error in the classification of a word of this sentence. This observation is used to detect and correct classification errors. For example in the sentence:

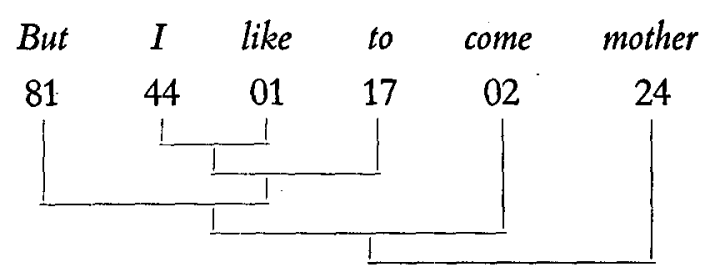


the word come had been classified in a wrong group 02 (indicative of intransitive verbs). When corrected $(22=$ infinitive of intr. verbs) we obtain the following structure:

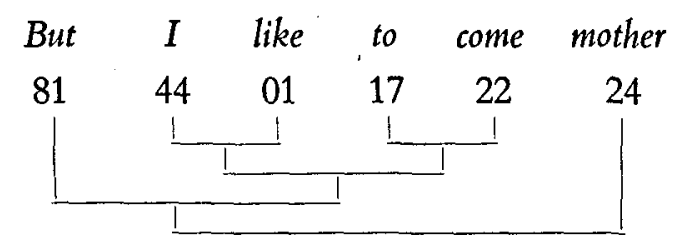

Another way to check the classification of words into groups is to use the quantity of information associated to the law of succession of two consecutive words. It is known from communication theory that the average amount of information by word is reduced when we know the law of succession of two consecutive words. This reduction is precisely equal to the average degree of correlation of all the groups:

$$
C=\sum_{i j} P_{i j} C_{i j}
$$

We shall call it quantity of information associated to the law of succession of two consecutive words.

In order to check the validity of the choice of the grammatical group for a word, the quantity of information associated to the law of succession of the groups is measured. Then, changing the choice of the group, the quantity of information is measured again for this new classification. The greater the quantity of information associated to a law of succession of the groups, the better the distribution of these words into these groups.

\section{CONCLUSION}

The sample chosen here (a novel by S. Maugham of 3500 words) is too short to obtain significant frequencies for the different structures.

This sample contains 200 sentences of an average length of 17 words.

In spite of the simplicity of the method of analysis employed, 72 sentences of an average length of 10 words have been correctly analysed.

This shows that the correlation of 2 consecutive words, although insufficient, will play an important part in the more elaborated methods of analysis that we are now developing. 
TABLE 1.

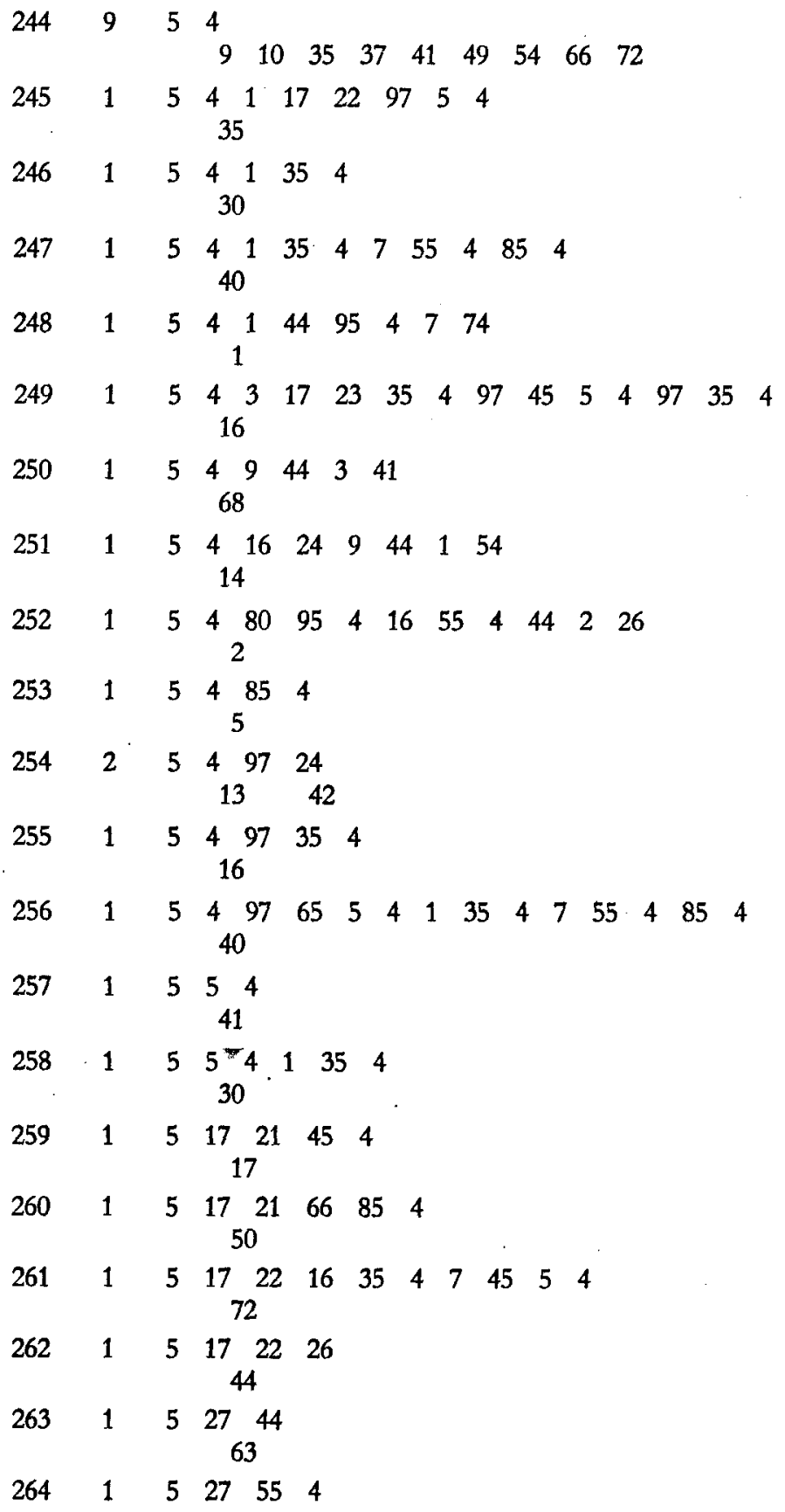




\section{TABLE 2.}

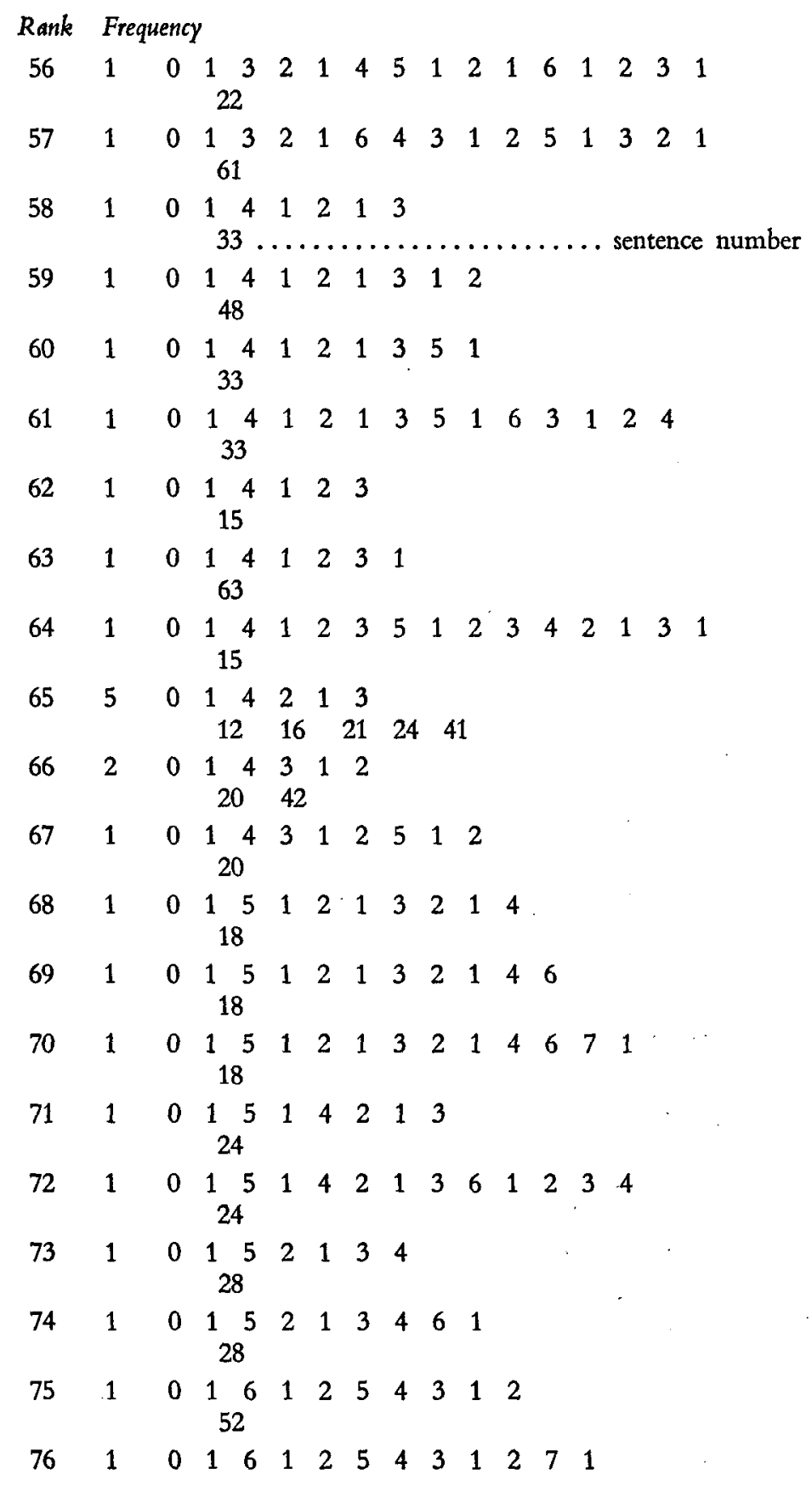


SENTENCE NO 60

\begin{tabular}{|c|c|c|}
\hline 3348 & & 65 \\
\hline & 1.421 & 1 \\
\hline 3349 & & 4 \\
\hline & 0.268 & 3 \\
\hline 350 & & 3 \\
\hline & 1.873 & 1 \\
\hline 351 & & 36 \\
\hline 352 & 1.804 & ${ }^{2}$ \\
\hline
\end{tabular}

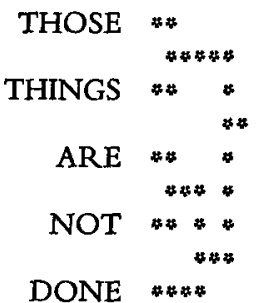

SENTENCE NO 56

\begin{tabular}{|c|c|c|}
\hline 3255 & & 29 \\
\hline & 4.345 & 1 \\
\hline 3256 & & 3 \\
\hline & 1.121 & 2 \\
\hline 3257 & & 5 \\
\hline & -0.095 & 3 \\
\hline 3258 & & 7 \\
\hline & 2.026 & 2 \\
\hline 59 & & 55 \\
\hline & 2.173 & 1 \\
\hline
\end{tabular}

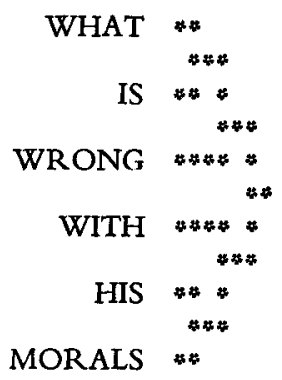

SENTENCE NO 58

\begin{tabular}{|c|c|c|}
\hline 3323 & & 54 \\
\hline & 2.306 & 1 \\
\hline 3324 & & 2 \\
\hline & 1.570 & 2 \\
\hline 3325 & & 66 \\
\hline & 2.689 & 1 \\
\hline 3326 & & 5 \\
\hline & -2.392 & 3 \\
\hline 327 & & 1 \\
\hline & 1.663 & 1 \\
\hline
\end{tabular}

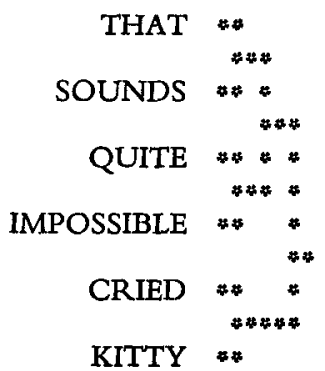


SENTENCE NO 36

\begin{tabular}{|c|c|c|}
\hline 1839 & & 44 \\
\hline 40 & 2.564 & 1 \\
\hline 340 & 1.859 & 2 \\
\hline 1841 & & 55 \\
\hline & 2.173 & 1 \\
\hline 1842 & & 4 \\
\hline 1843 & 1.012 & 3 \\
\hline & 1.180 & 2 \\
\hline 44 & 1.573 & $1^{45}$ \\
\hline
\end{tabular}

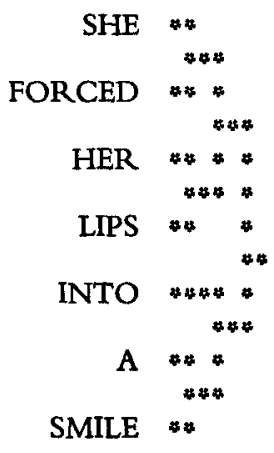

SENTENCE NO 20

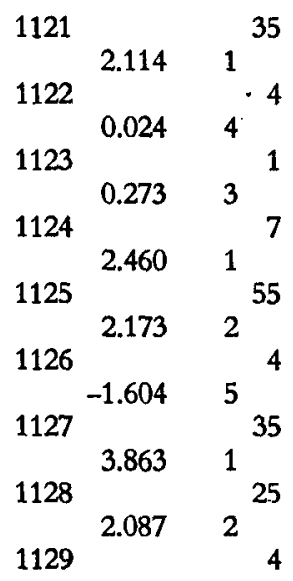

THE =

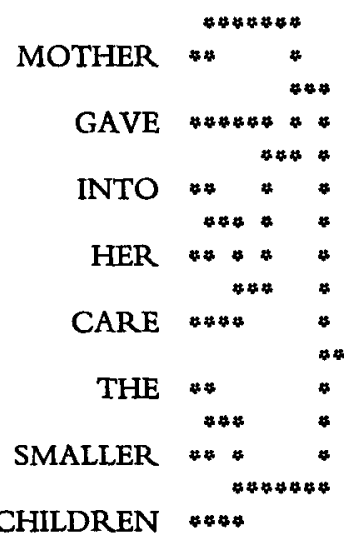


SENTENCE NO 19

\begin{tabular}{|c|c|c|}
\hline 1079 & & 24 \\
\hline & 1.644 & 1 \\
\hline 080 & & 1 \\
\hline & 0.926 & 2 \\
\hline 1081 & & 35 \\
\hline 82 & 2.114 & 1 \\
\hline & -1.198 & 4 \\
\hline 1083 & & 45 \\
\hline & 1.573 & \\
\hline 1084 & & 3 \\
\hline 1085 & 0.221 & ${ }^{3} 87$ \\
\hline & 2.511 & \\
\hline 1086 & & 55 \\
\hline & 2.173 & 2 \\
\hline
\end{tabular}

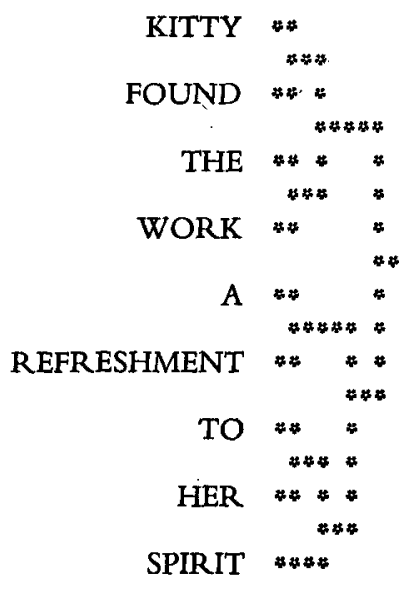

SENTENCE NO 49

\begin{tabular}{llll}
2820 & \multicolumn{3}{c}{44} \\
2821 & 2.564 & 1 & \\
& 0.273 & 3 & \\
2822 & & & 7 \\
& 2.460 & 1 & \\
2823 & & & 55 \\
& 2.173 & 2 & \\
2824 & & & 4 \\
& -5.241 & 4 & \\
2825 & & & 4 \\
2826 & 1.012 & 1 & \\
2827 & -0.801 & 2 & \\
& 1.860 & 1 & \\
2828 & & & 4
\end{tabular}

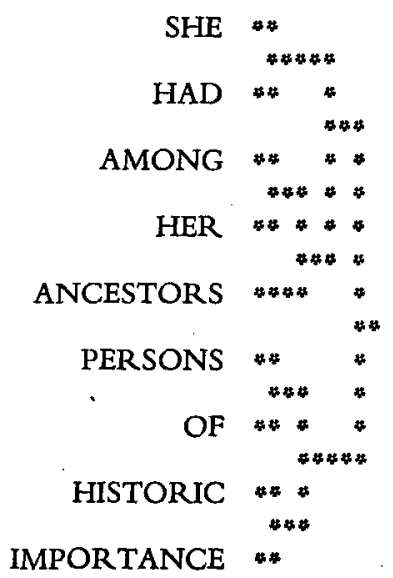


SENTENCE NO 50

\begin{tabular}{|c|c|c|}
\hline 830 & & 94 \\
\hline & 2.047 & 4 \\
\hline 831 & & 68 \\
\hline & 5.493 & 2 \\
\hline 332 & & 28 \\
\hline & 7.456 & 1 \\
\hline 333 & & 33 \\
\hline & 2.742 & 3 \\
\hline 834 & & 5 \\
\hline & 0.490 & 5 \\
\hline 2835 & & 17 \\
\hline & 4.406 & 1 \\
\hline 836 & & 21 \\
\hline & 0.540 & 3 \\
\hline 2837 & & 66 \\
\hline & 0.870 & 2 \\
\hline 38 & & 85 \\
\hline & 2.194 & 1 \\
\hline
\end{tabular}

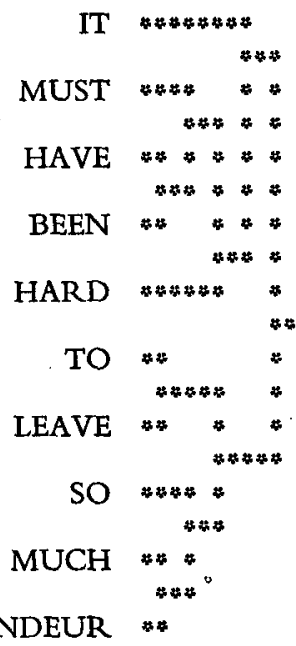

SENTENCE NO 52

\begin{tabular}{|c|c|c|}
\hline 2850 & & 44 \\
\hline & 2.564 & 1 \\
\hline & 0.926 & 6 \\
\hline 2852 & & 35 \\
\hline & 3.863 & 1 \\
\hline 2853 & & 25 \\
\hline & 2.087 & 2 \\
\hline 2854 & & \\
\hline & 1.192 & 5 \\
\hline 2855 & & 9 \\
\hline & 2.438 & 4 \\
\hline 2856 & & 44 \\
\hline & 2.727 & 3 \\
\hline 2857 & & 8 \\
\hline & 3.709 & 1 \\
\hline 2858 & & 6 \\
\hline & 2.804 & 2 \\
\hline 2859 & & 31 \\
\hline & -0.655 & 7 \\
\hline 2860 & & \\
\hline & 1.663 & 1 \\
\hline
\end{tabular}

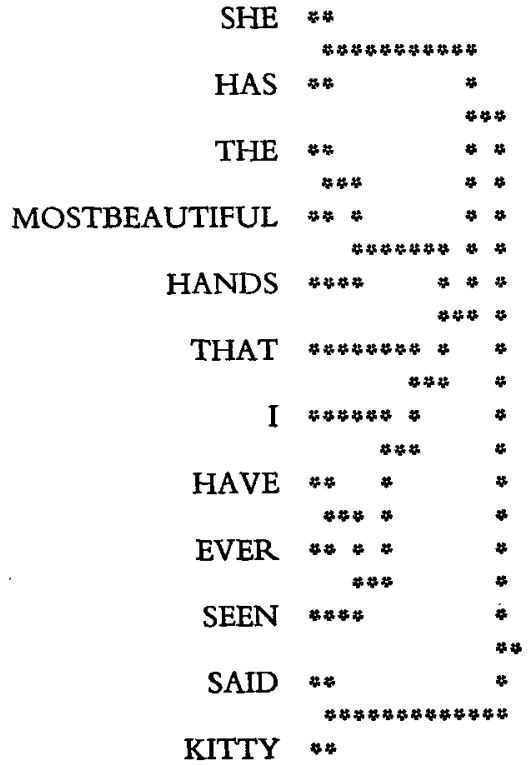


SENTENCE NO 55

\begin{tabular}{|c|c|c|}
\hline 3216 & & 80 \\
\hline & 2.061 & 1 \\
\hline 217 & & 46 \\
\hline & 0.265 & 2 \\
\hline 3218 & & 24 \\
\hline & 3.245 & 1 \\
\hline 3219 & & 2 \\
\hline & -0.934 & 5 \\
\hline 3220 & & 44 \\
\hline & 2.564 & 1 \\
\hline 3221 & & 1 \\
\hline & 1.125 & 2 \\
\hline 3222 & & 6 \\
\hline & 2.227 & 1 \\
\hline 3223 & & 56 \\
\hline & 0.505 & 3 \\
\hline 3224 & & 17. \\
\hline & 4.406 & 1 \\
\hline 3225 & & 21 \\
\hline 226 & 1.036 & ${ }^{2}$ \\
\hline & 0.302 & 4 \\
\hline 227 & & 22 \\
\hline
\end{tabular}

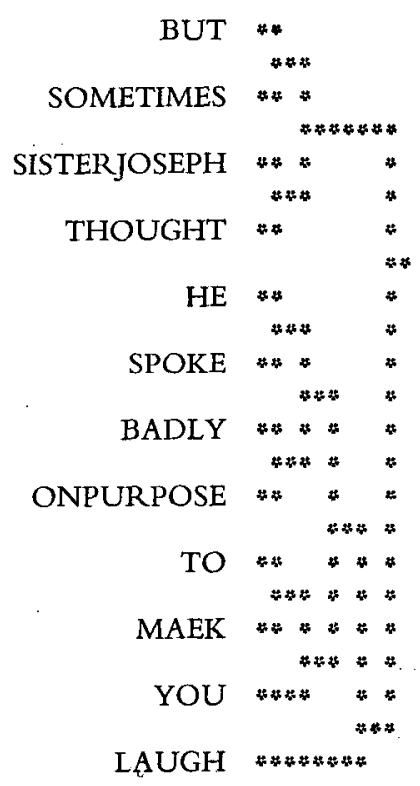


SENTENCE NO 32

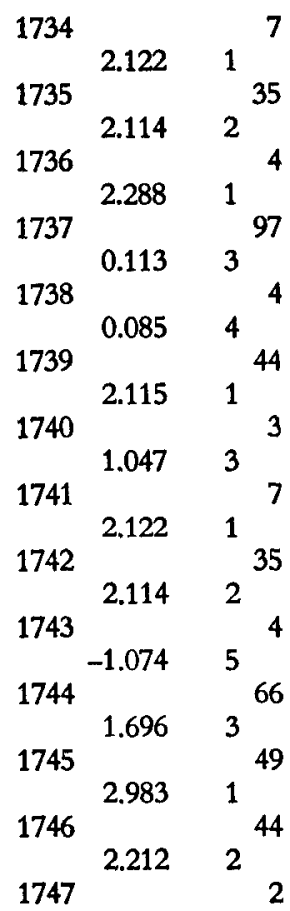

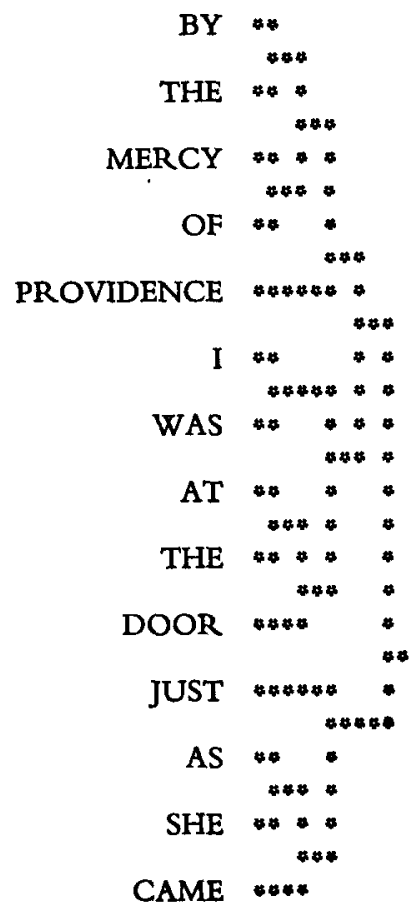


SENTENCE NO 14

\begin{tabular}{|c|c|c|}
\hline 728 & & 94 \\
\hline & 3.529 & 1 \\
\hline 29 & 1.873 & 2 \\
\hline 30 & & 36 \\
\hline & 1.760 & 3 \\
\hline 31 & & 49 \\
\hline & 2.983 & 1 \\
\hline 732 & & 44 \\
\hline & 2.564 & 2 \\
\hline 33 & & 1 \\
\hline & 1.232 & 4 \\
\hline 34 & & 45 \\
\hline & 2.379 & 1 \\
\hline 5 & & 5 \\
\hline & 1.860 & 2 \\
\hline 36 & & 4 \\
\hline & 1.777 & 3 \\
\hline 37 & & 16 \\
\hline & 2.637 & 1 \\
\hline 73 & & 24 \\
\hline 739 & 0.075 & $\begin{array}{l}59 \\
{ }^{5} 9\end{array}$ \\
\hline & 2.438 & 2 \\
\hline 740 & & 44 \\
\hline & 2.564 & 1 \\
\hline & & \\
\hline & 1.710 & 54 \\
\hline
\end{tabular}

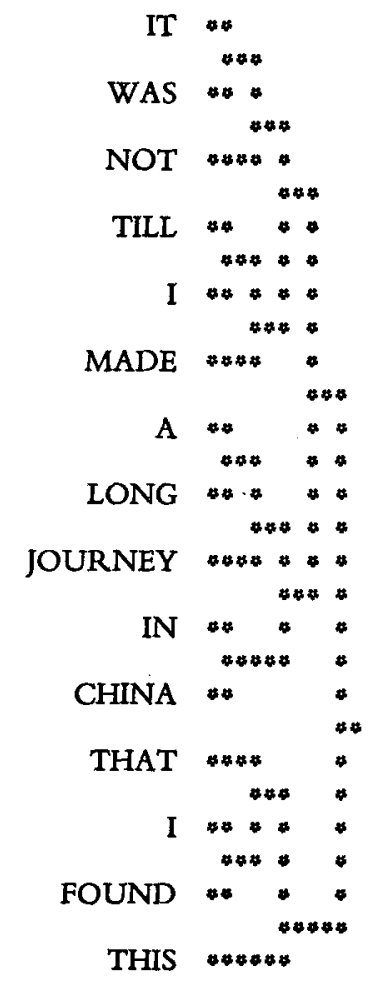


SENTENCE NO 15

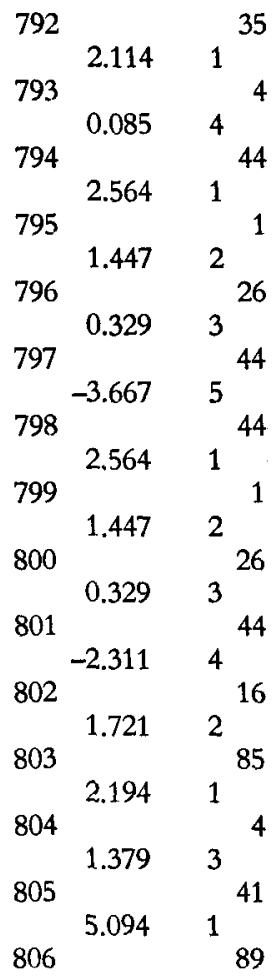

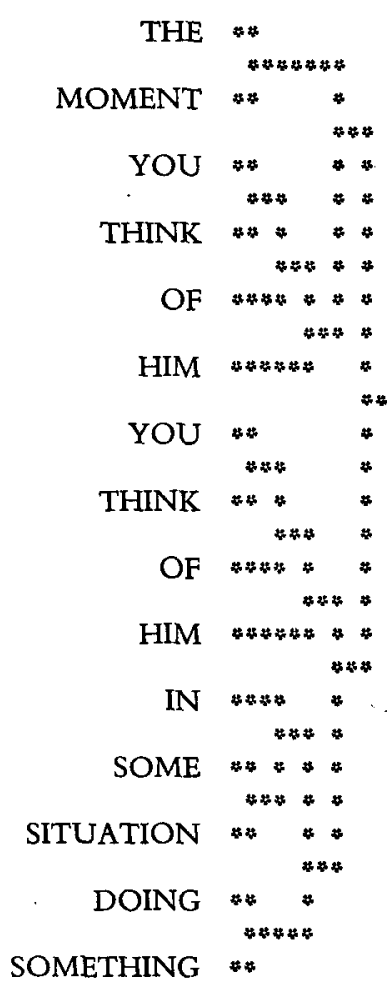


SENTENCE NO 22

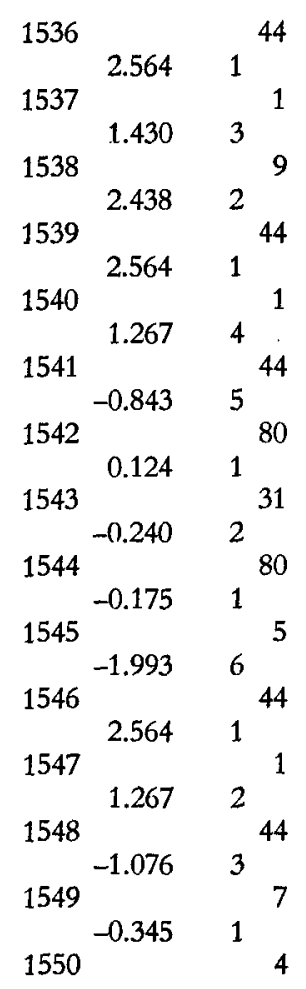

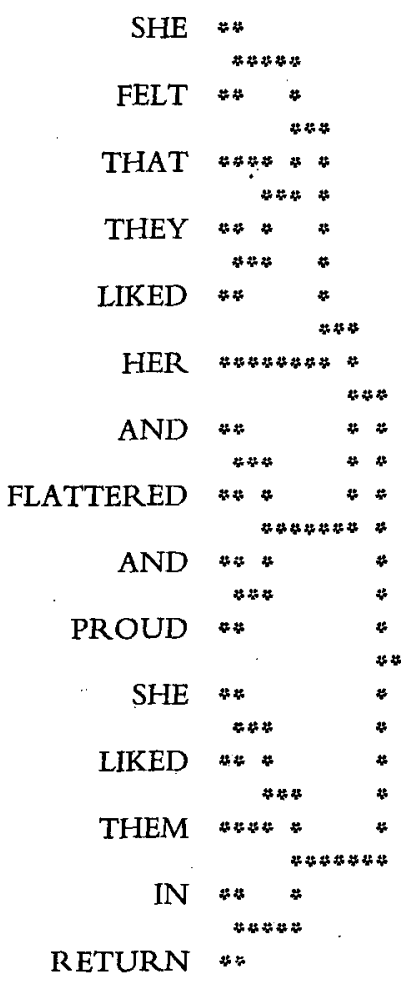


TABLE 3.

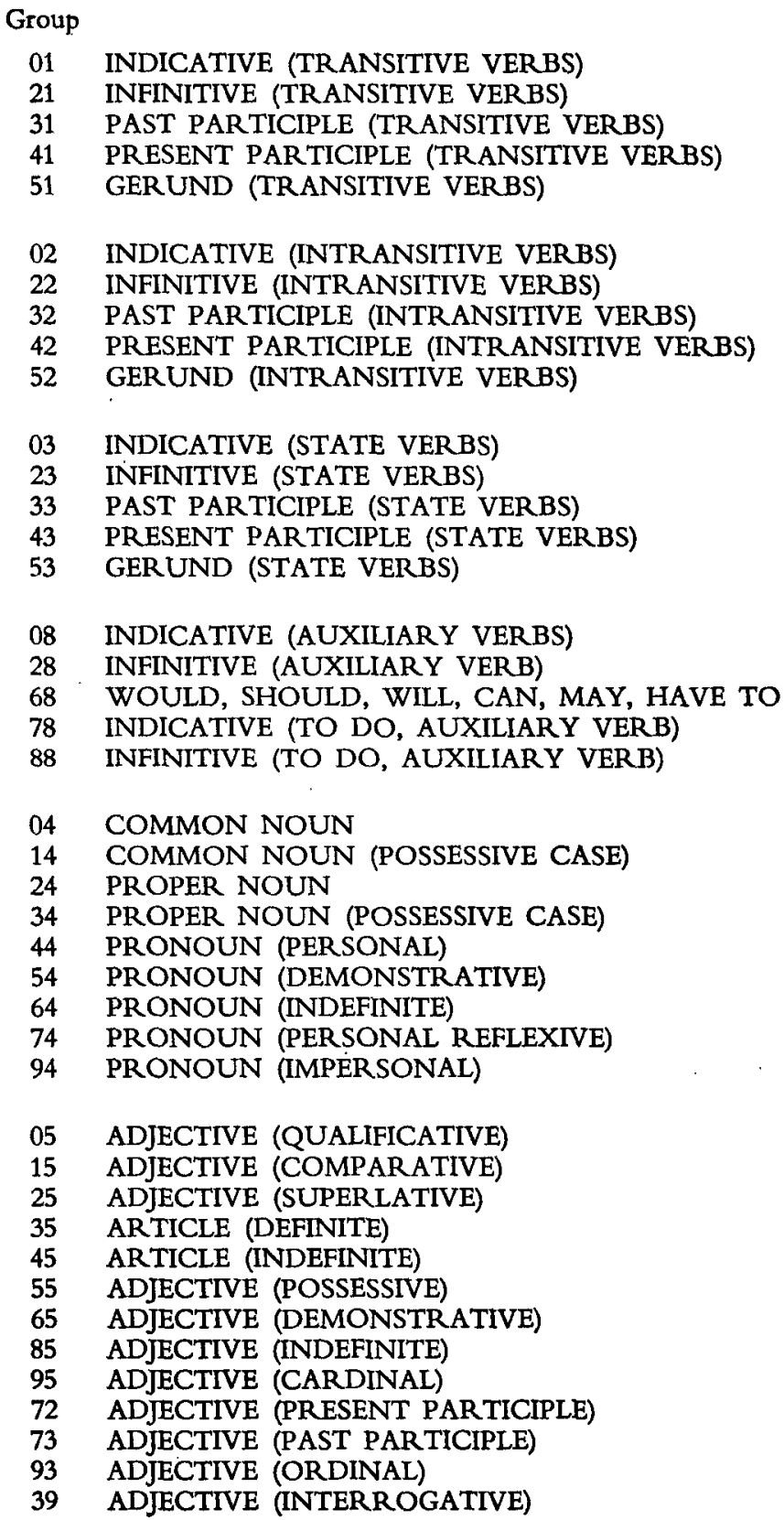




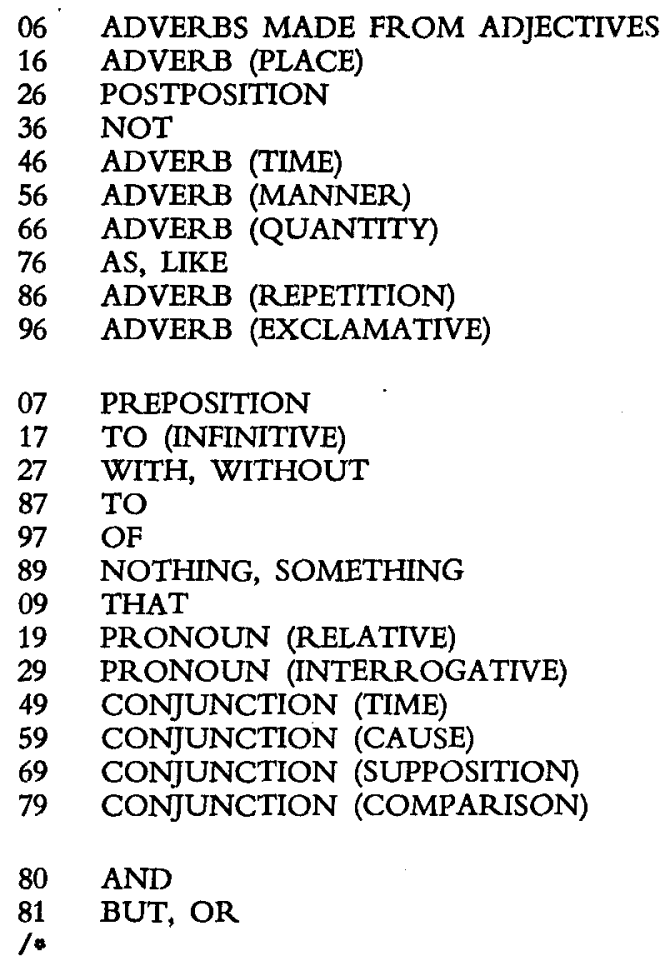


